\title{
Neutral wind effects on ion outflow at Mars
}

\author{
L. Andersson ${ }^{1}$ and R. E. Ergun ${ }^{1,2}$ \\ ${ }^{1}$ Laboratory of Atmospheric and Space Physics, University of Colorado, Boulder, CO 80303, United States \\ ${ }^{2}$ Department of Astrophysical and Planetary Sciences, University of Colorado, Boulder, CO 80302, United States
}

(Received February 9, 2011; Revised May 18, 2011; Accepted June 23, 2011; Online published March 8, 2012)

\begin{abstract}
This paper evaluates the influence of neutral winds on ions using the CAPIT (Combined Atmospheric Photochemistry and Ion Tracking) code, which includes ionospheric photochemistry and plasma wave energization. Based on two different wind profiles, ion outflow is, to first order, not sensitive to neutral winds. However, the ion density profiles are clearly affected by neutral winds at the exobase. For example, the direction of a strong neutral wind will dramatically change the location of the highest ion densities. This finding suggests that strongly varying neutral winds at the exobase complicate the analysis of in-situ observations. To evaluate dynamic effects of the different magnetic field configurations, crustal fields are included in the simulation domain. The outflow of $\mathrm{O}_{2}{ }^{+}$ions is the most dynamic, partly because the photochemical production is almost a factor of two larger than the photochemical loss so loss to space is important (for other ions, this ratio is closer to one and loss to space is small compared to photochemical production). Since the photochemical production rate of $\mathrm{O}_{2}{ }^{+}$is relatively slow, it takes time to replenish $\mathrm{O}_{2}{ }^{+}$that is lost to space, so loss to space over long periods should be relatively constant.
\end{abstract}

Key words: Double layer, aurora, acceleration.

\section{Introduction/Background}

Understanding how surface water was lost at Mars is still an important unsolved problem. One possibility is that water retracted into the ground, but this loss mechanism is yet to be demonstrated. It is therefore of interest to understand how water (i.e. $\mathrm{O}$ and $\mathrm{H}$ ) in the atmosphere can be lost to the solar wind. Once in the solar wind, ions and neutrals (neutral which will eventually be ionized by solar EUV) are transported away from the planet. Therefore the transport of both ions and neutrals from the top-side of the ionosphere to the solar wind is an important regulator of the outflow rate. In this paper the focus is on ion transport between the top-side of the atmosphere to higher altitudes.

A number of studies have invoked atmospheric codes, which have modeled Mars's ionosphere and ion production well (such as Nair et al., 1994; Haider, 1995; Fox, 1997; Fox and Yeager, 2006; Lammer et al., 2003). However, most of these studies rely on the only electron temperature profile we have from Mars, the Viking Landers measurements, and often do not include any energy source from the local plasma (solar wind/plasma waves). These codes represent the ion production to some degree but ultimately do not include important processes that energize the ions and lead to escape.

There also have been a high number of studies on how ions, once generated close to the exobase, are transported into the solar wind (such as Ma et al., 2004; Modolo et al., 2005; Brecht and Ledvina, 2006; Ma and Nagy, 2007;

Copyright (c) The Society of Geomagnetism and Earth, Planetary and Space Sciences (SGEPSS); The Seismological Society of Japan; The Volcanological Society of Japan; The Geodetic Society of Japan; The Japanese Society for Planetary Sciences; TERRAPUB.

doi:10.5047/eps.2011.06.047
Terada et al., 2009; Kallio et al., 2010). Many of these studies assign the ion densities and the temperatures close to the exobase. However, the limiting processes of ion outflow may be ion production and ion energization, which these codes often do not include. Therefore, in earlier work by Andersson et al. (2009), the Combined Atmospheric Photochemistry and Ion Tracing (CAPIT) code was developed to understand how the ion production and ion energization controls the total ion outflow. The CAPIT code includes ion production due to photochemical production and ion heating through plasma waves heating.

The best in-situ observations of the Mars ionosphere come from the Viking Landers density and electron temperature profiles (Hanson et al., 1977; Hanson and Mantas, 1988). An understanding of the composition of the out flowing ions and the total ion outflow can be derived from observation by several satellite missions (Nairn et al., 1991; Carlsson et al., 2006; Dubinin et al., 2006; Lundin et al., 2009). Observations have demonstrated that ion outflow is modulated by solar wind forcing (Nilsson et al., 2010) and that particle fluxes are modulated at ion cyclotron frequencies down at altitudes of $\sim 300 \mathrm{~km}$ (Winningham et al., 2006) and at longer time frames (Duru et al., 2008). At $\sim 300 \mathrm{~km}$ altitudes, strong fluxes of energetic ion conics have been observed (Lundin et al., 2006). Lastly, magnetic fluctuations near the $\mathrm{O}^{+}$cyclotron frequency close to $300 \mathrm{~km}$ have been observed (Espley et al., 2004), but electric field measurements at these frequencies have not been made in the low-altitude ionosphere.

The work presented here differs from other studies in that the primary energy input into the ions is assumed to come from plasma waves. Induced electric fields from the solar wind are not included because, to first order, they act 
uniformly on all ions (lift all ions up on one hemisphere and suppress them on the other). Furthermore, the size of the induced fields are not precisely known, but expected to be less than the electric field associated with the waves. There are no other electric fields other than the waves in this work. For example, an ambipolar electric field is not included in this work. The heating rates of the plasma waves are assumed to be those of ion cyclotron waves since such waves have been seen in the magnetic signatures.

In recent work by Valeille et al. (2009), the magnitude and direction of the neutral winds are studied with the Mars Thermospheric General Circulation Model (MTGCM). The formulation of this model, along with some of its applications and validation studies are summarized in Bougher et al. (2008). The neutral winds used in this paper are based on the peak speeds and directions suggested by Valeille et al. (2009).

The paper presents the CAPIT code in Section 2 and the simulation set up. The simulation result is presented in Section 3 and a summary is given in Section 4.

\section{Simulation Setup}

The analysis of the neutral wind's effect on ion outflow is analyzed with the CAPIT code (Andersson et al., 2009). The code is two-dimensional. Photochemical reactions occur on a grid. Ions are created on a statistical basis based on reaction rates. After ions are created, they are individually traced as macro-particles under electromagnetic and gravity forces. The macro-ions are heated by waves imposed from the top of the simulation domain and cooled down by collisions in the dense atmosphere at lower altitudes. The ion species that are followed are $\mathrm{N}_{2}{ }^{+}, \mathrm{O}_{2}{ }^{+}, \mathrm{O}^{+}$, and $\mathrm{CO}_{2}{ }^{+}$.

The simulation domain is \pm 45 degrees from the subsolar point along the equator (Fig. 1). The altitude range is from $160 \mathrm{~km}$ to $460 \mathrm{~km}$, such that the exobase is within the simulation domain. The location of the so-called ionopause at Mars varies; often observed between $300-500 \mathrm{~km}$ of altitude (Mitchell et al., 2001). This boundary is assumed to be just outside the simulation domain.

The photochemical reactions are based on fixed neutral density profiles (versus altitude), that is, the change of the neutral densities by the photochemical reactions is assumed to be negligible. The neutral profiles are derived from the dusk measurements from the Viking Landers (Nier and McElroy, 1977). The observed neutral densities by the Viking Landers at the flanks is expected to be lower than the densities at noon due to solar zenith angle effects. In the simulation domain, the neutral densities at $\pm 45^{\circ}$ are assumed to be the same as the Viking observations. The density increases toward noon as function of the solar zenith angle ( $1 / \cos )$. More sophisticated neutral profiles, as function of solar zenith angle, are not needed for the questions addressed in this paper. The chemical reaction rates are given in Andersson et al. (2009) and the ionization are rates assumed for quiet sun conditions.

Ionospheres are quasi-neutral, so the electron density is derived from the sum of the ion densities. An ion, when created, is initially at the same temperature as the neutral species. The ion temperatures are re-calculated in the simulation as the ions are heated by waves and/or cooled by

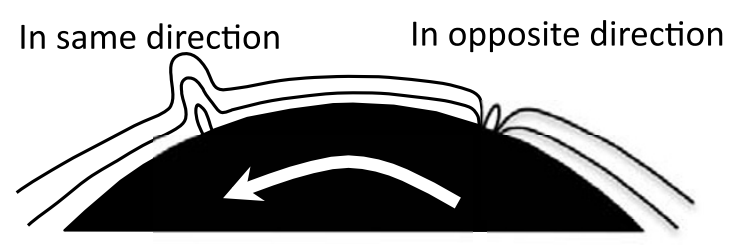

Fig. 1. An Illustration of the draped magnetic field $\pm 45^{\circ}$ along the Mars surface and two crustal magnetic fields. One crustal field is directed with (same) the same direction as the draped magnetic field; the second crustal field is directed one against (opposite). The arrow indicates the rotation direction of Mars as seen from north pole.

collisions. The electron temperature is not directly calculated. It is assigned a value the value of the average ion temperature $20 \mathrm{~km}$ below in altitude, making them slightly cooler.

In the code, collisions are based on cross sections with the neutrals. In an ion-neutral collision, the momentum of the ion is transferred with random impact parameter and velocities so that the ion temperatures and drifts approaches the neutral atmosphere values. The atmosphere is drifting at the neutral wind velocity. Zero neutral wind corresponds to the sub-solar point (i.e. not co-rotating with the planet).

The magnetic field is assumed to have a flared topology (as in Andersson et al., 2009) with the addition of crustal fields. A crustal field is modeled as dipole field rotating with the surface of the planet. The crustal fields can be orientated with or against the draped magnetic field as illustrated in Fig. 1.

Wave energy flux is introduced at the high-altitude boundary of the simulation domain. The initial magnitude of the wave power is the same for all simulations in this paper. As the wave energy flux propagates downward, the ions are heated and the wave energy flux decreases. With the set levels of wave power, nearly all energy is deposited into the ions. A significant part of the wave energy is deposited into the neutrals due to ion-neutral collisions. The collision rate at the low-altitude boundary of the simulation domain is such that the ions have the same temperature and drift as the neutrals.

The ion loss is quantified using two methods: (1) all the particles leaving the simulation domain above a selected altitude and (2) a subset of those particles that have reached escape velocity. In the first method, particles leaving the simulation domain are assumed to be lost since they are magnetized and the draped magnetic field will guide them into the tail away from the planet where plasma processes can energize them further. With the second method, the particles with speeds above escape velocity will be lost as long as they have a component of their velocity that is away from the planet. In addition to recording the number of particles leaving, the age of the individual macro particles in the simulation domain is also stored.

Two different neutral wind profiles are evaluated (Fig. 2). The peak speed of the winds are based on the Valeille et al. (2009) results while the altitude profile is varied for understanding at which altitude the neutral winds have the strongest influence on the ion distributions. Wind A repre- 

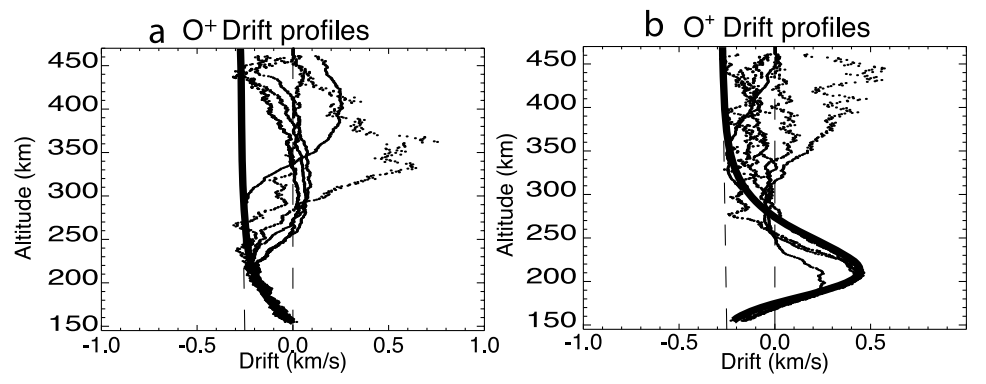

Fig. 2. The two wind profiles used in this paper (thick black solid line). Wind A (panel a) is the result of winds canceling the co-rotation drift (dashed line at $\sim-350 \mathrm{~m} / \mathrm{s}$ ) resulting in almost no wind at $150 \mathrm{~km}$ and co-rotation at high altitudes. Wind B (panel b) has strong winds peaking around 200 $\mathrm{km}$ resulting in an opposite and slightly stronger drift compared to the co-rotation speed at that altitude. The dotted-lines represent different drift profiles (as a function of position along surface) for $\mathrm{O}^{+}$ions when the simulation has reach steady state.

sents a stagnant flow at low altitudes $(0 \mathrm{~km} / \mathrm{s}$ with respect to subsolar point) and co-rotation at high (moving with the surface, $\sim-0.2 \mathrm{~km} / \mathrm{s}$ ). The magnitude of Wind $\mathrm{B}$ is stronger than that of Wind A and oppositely directed with respect to the planet's rotation near the exobase. Below and above the exobase Wind B flow is set to co-rotation speed. This particular profile may be unrealistic, but the results reveal that the winds at the exobase have the strongest influence on the ion distributions. At high altitudes (well above the exobase), the ion production rate and collision rate is low resulting in little effect from neutral winds. At low altitudes (below the exobase), the collision frequency is high, so the ions move with the neutral wind and few escaping ions. Neutral winds well below the exobase have less influence on ion outflow.

In previous work, the impacts of wave power and ion production rate (ionization rates) on ion outflow rates were studied (Andersson et al., 2009). The ion outflow rate was fairly linear in response to the amount of available wave power until the ions started to lose their energy to the neutrals at lower altitudes, i.e. wave power ended up in the neutrals. The optimum location at which to heat the ions is where the ion density is high but the ion-neutral collision frequency is small enough that significant energy is not transferred to the neutrals. If the energy deposition is concentrated at this location, the ion heating and subsequent outflow is efficient. To the contrary, if the energy deposition is spread out, ion heating is less efficient. For strong outflow, enough energy must be deposited into the same ion and result in the loss of that ion rather than at less efficient altitudes where the energy deposition is evenly spread out such that all ions gain some energy but not enough to escape. It was concluded that both wave power and ionization rates are important for today's outflow rates. Neither of the two parameters resulted in a non-linear response in the outflow. It is therefore difficult, based on the present knowledge of parameters, to suggest that either wave power or ionization rates can alone explain the expected loss of water at Mars in the past.

\section{Simulation Results}

The predicted $\mathrm{O}^{+}$and $\mathrm{O}_{2}{ }^{+}$densities in draped magnetic field topology for the two different wind profiles are presented in Fig. 3. The density is represented by the color, the $x$-axis is distance along surface and altitude is represented by the $y$-axis. The title on the $y$-axis indicates which wind and which time each row represents. Time $0 \mathrm{~h} 0 \mathrm{~min}$ starts with a draped magnetic field (panels a to d). Times $10 \mathrm{~h} 50$ min and $21 \mathrm{~h} 45 \mathrm{~min}$ (panels e to 1) include crustal fields with the draped magnetic field and are discussed later.

It is clear from the figures that the ion density altitude profiles is affected by exobase ( $\sim 250 \mathrm{~km}$ in altitude) neutral winds. The ion densities at higher altitudes show the least change with dramatically differing wind patterns. From multiple runs with different wind profiles, we find that neutral winds well below and well above the exobase are less important. The ion production and heating near the exobase is most sensitive to the neutral wind velocity. Below the exobase, the high collision rates cause a rapid loss before the plasma waves cause any significant heating. Near the exobase, the ion heating has time to effect the ion temperature and loss rate on a time scale that is faster than the reabsorption time. Above the exobase, ion production rates are small. With the parameters used in the simulations, the wind strength and direction are mainly important in the altitude range of $180-240 \mathrm{~km}$.

The ion distribution functions also are influenced by the neutral winds. This effect is shown in the ion velocityvelocity distributions that are presented in Fig. 4. At locations with high densities the ion distributions are cold (i.e. mainly at low altitudes and on the flank which the neutral wind is blowing towards, such as panel b and g). Heated ion distribution in the form of ion conics can be found in low-density regions and where the magnetic mirror force is important (i.e. high altitudes such as panel $\mathrm{d}$ and $\mathrm{j}$ ). The draped magnetic field is such that the magnetic gradient is strong enough to direct the heated ions away from the subsolar point at altitudes above the exobase. This high altitude outflow is mostly independent of the neutral wind pattern (the doted lines for the morning flank flows in Fig. 2 has positive flow at high altitude compare to evening flows which is negative).

The average age of the particles in the simulation can be inferred by looking at distributions presented in Fig. 4. Near the exobase the lifetime of $\mathrm{O}^{+}$and $\mathrm{O}_{2}{ }^{+}$is short, $<15$ minutes, whereas $\sim 50 \mathrm{~km}$ above the exobase the average lifetime can be long, $>40$ minutes. It is in the older populations that the heating clearly is visible. At low latitudes, since the particles are younger and have not been heated significantly the ion drift speeds can be used to infer the neutral wind speed and direction around the exobase. 

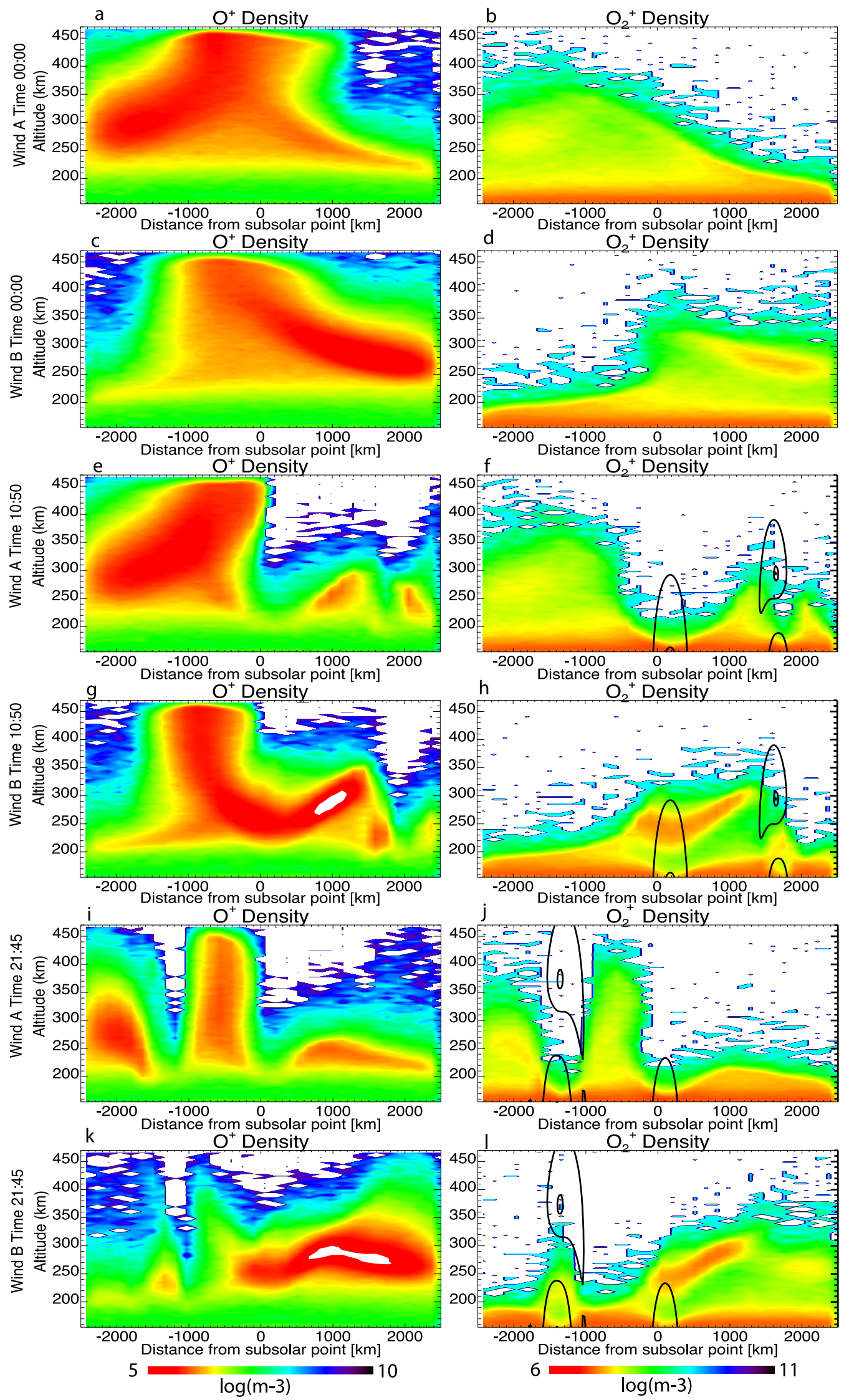

Fig. 3. $\mathrm{O}^{+}$and $\mathrm{O}_{2}{ }^{+}$density maps for two different winds and three different magnetic field configurations. The density maps (color) are presented as function of distance along surface ( $x$-axis) and altitude ( $y$-axis). Left $y$-title indicates which wind profile and which time are plotted. Time $0 \mathrm{~h} 0$ min (panels a to d) represent a static magnetic field with only draped magnetic field present. Times $10 \mathrm{~h} 50 \mathrm{~min}$ and $21 \mathrm{~h} 45 \mathrm{~min}$ (panels e to l) are associated with the times given in Fig. 5 and explained in Section 3. In the right column the location of the crustal fields are indicated by the black contour lines. 

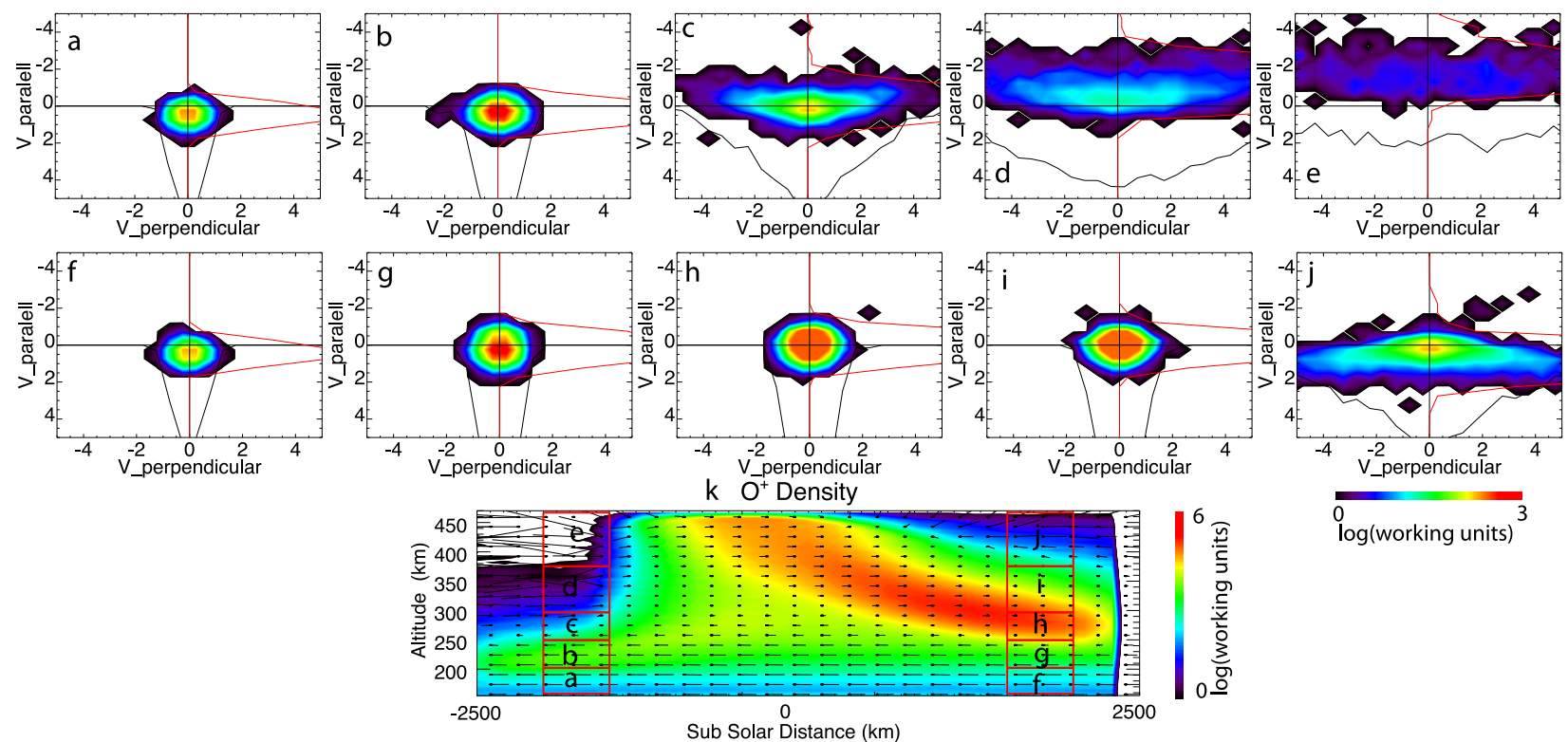

log(working units)

Sub Solar Distance $(\mathrm{km})$

Fig. 4. $\mathrm{O}^{+}$velocity-velocity distributions for five different altitudes at two different surface locations as indicated by the panel labels located in the white boxes in the density map panel $\mathrm{k}$. Also in panel $\mathrm{k}$ is the wind direction identified with flow vectors (the start of the vector is located at the dot). At the low density location the velocity direction is less reliable. In the example presented here Wind B is used.

The main pattern that emerges from analyzing several simulations with different wind profiles is that the ion distributions at high densities are cold. That is, it is at the high altitudes, where the low-density regions are located, heated flowing ions are observed. This same behavior has been observed at Earth. The low density regions have the strongest ion outflows flows and the main energization region is at the boundary between the high density regions and low density regions.

The crustal fields play an important role since they can lift ions to high altitudes where they can experience longer heating times, or lower the ions where they can be reabsorbed. The simulation was run for long periods during which three regions of crustal magnetic field crossed the simulation domain (mimicking Mars' rotation) as indicated in Fig. 1. The three regions of crustal fields were separated far enough that at most one crustal field was within the simulation domain at any given time (with a co-rotation speed of $\sim 0.2 \mathrm{~km} / \mathrm{s}$ a crustal field moves across simulation domain in $\sim 20$ minutes but the effect of the field is noticeable much longer).

The first crustal field region contained one single dipole field directed with the draped magnetic field direction. The second region contained two dipoles next to each other. The first dipole was stronger than the second and the first was directed with the draped field while the second against. The third region contained two dipoles with the first directed against the draped field followed by the second directed with the draped magnetic field. The $\mathrm{O}^{+}$and $\mathrm{O}_{2}{ }^{+}$density maps from two times during this 24-hour period and from two different wind profiles are presented in the four lowest rows in Fig. 3. Panels e to $h$ are from a time when the second crustal field region is passing (time $10 \mathrm{~h}$ and $50 \mathrm{~min}$ ) and panels $i$ to 1 are from the third crustal field region passing (time $21 \mathrm{~h}$ and $45 \mathrm{~min}$ ). Comparing all three different magnetic field scenarios presented in Fig. 3 it is clear that neutral winds can redistribute the ions so much that the high/low density regions in longitudinal direction are completely in different locations for different winds, most clearly observable in $\mathrm{O}_{2}{ }^{+}$as a result of their long lifetime. The location of the crustal fields is marked with black contours in the panels for $\mathrm{O}_{2}{ }^{+}$densities.

The history of the respective outflows as a function of time is presented in Fig. 5. The first six panels (a through f) are for wind $\mathrm{A}$ and the same information for wind B is in the lower six panels ( $g$ through 1 ). For each panel, an outflow quantity as a function of time is presented for $\mathrm{O}^{+}$, $\mathrm{O}_{2}{ }^{+}, \mathrm{CO}_{2}{ }^{+}$, and $\mathrm{N}_{2}{ }^{+}$(blue, green, red, and yellow lines respectively). The six panels represent the following: (a and $g$ ) the ion outflow based on all particles above a fixed altitude, ( $b$ and $h$ ) the ion outflow using only particles above the escape velocity above a fixed altitude, (c and i) the ratio between the ion production and loss within the simulation domain from photochemical reactions, $(\mathrm{d}$ and $\mathrm{j}$ ) the relative outflow based on the $\mathrm{O}^{+}$outflow rate using all particles, (e and $\mathrm{k}$ ) the relative outflow based on the $\mathrm{O}^{+}$outflow rate using only particles above escape velocity, and (f and 1 ) the fraction of escaping particles for each ion species that has reached escape velocity.

In contrast to the altitude profile differences for the two winds presented in Fig. 3, the ion outflow differences in Fig. 5 (panels a and $g$ and $b$ and $h$ ) are not significant. The outflow varies by a factor of $2-3$ as the three crustal field regions move through the simulation domain. The direction of the leading crustal field with respect to the draped magnetic field affects the temporal evolution of the ion outflow. The conclusion is therefore that, while neutral winds and crustal fields can produce strong, short-period, localized fluctuations in ion density and ion outflow, the neutral wind and crustal fields are not important for long-term ion outflow to first order. For time scales longer than the Mars rotation period, the crustal fields effect are unimportant. 

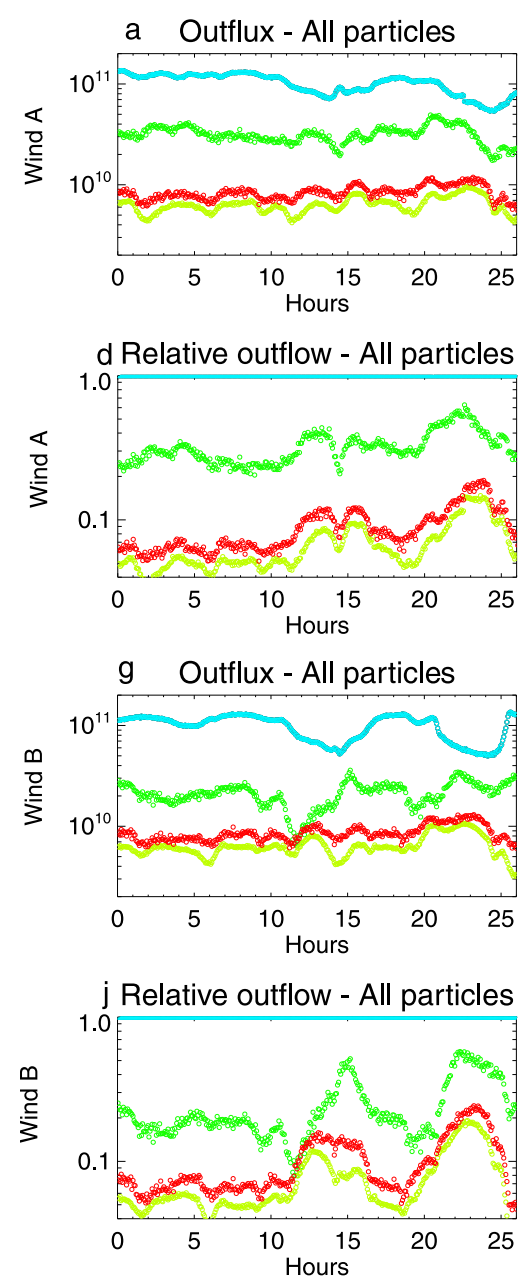

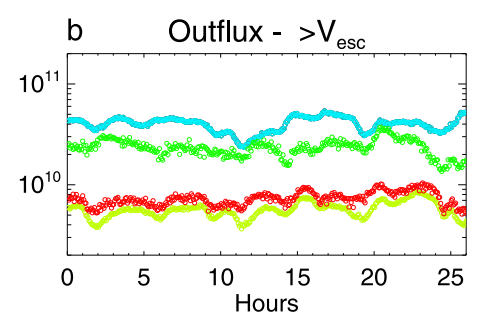

e Relative outflow - $>\mathrm{V}_{\text {esc }}$
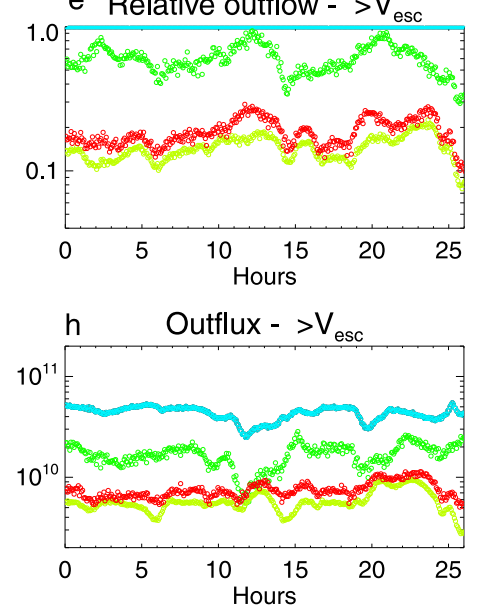

$\mathrm{k}$ Relative outflow - $>\mathrm{V}_{\mathrm{esc}}$

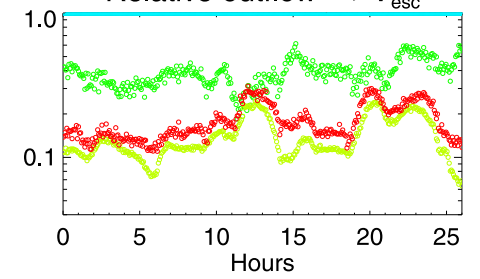

c Chemistry Production/Loss

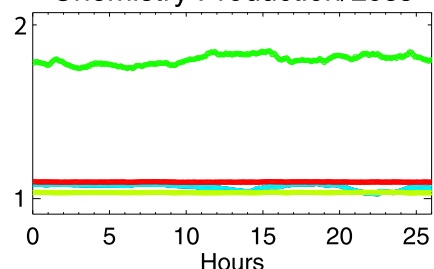

$f$ Relative portion - $>V_{\text {esc }} /$ All

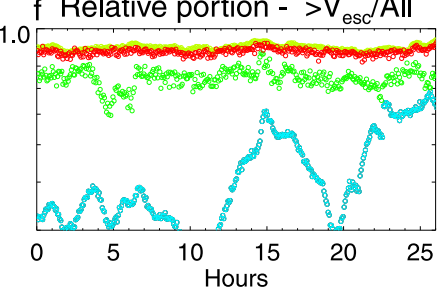

i Chemistry Production/Loss

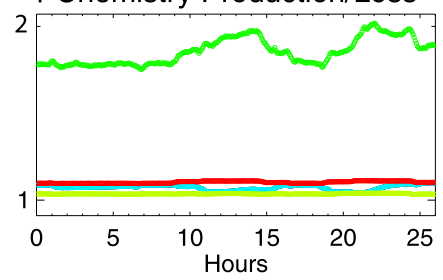

I Relative portion - > $\mathrm{V}_{\text {esc }} /$ All

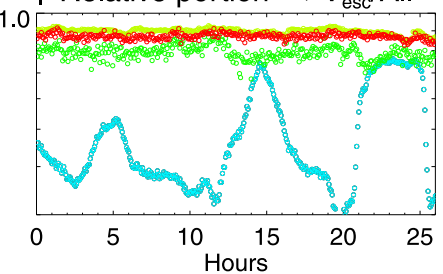

Fig. 5. The ion outflow characteristics as function of time for $\mathrm{O}^{+}, \mathrm{O}_{2}{ }^{+}, \mathrm{CO}_{2}{ }^{+}$, and $\mathrm{N}_{2}{ }^{+}$(blue, green, red, and yellow lines respectively). The six upper panels are from simulation run using Wind A and the six lower panels are from Wind B and all outflows are derived above a fixed altitude. The six pairs of panels represent, ( $\mathrm{a}$ and $\mathrm{g}$ ) outflow of all particles, (b and $\mathrm{h}$ ) out flowing particle above escape velocity, (c and i) the ratio between the ion production and loss within the simulation domain from photo-chemical reactions only, ( $\mathrm{d}$ and $\mathrm{j}$ ) the relative outflow of all particles normalized to $\mathrm{O}^{+}$, (e and $\mathrm{k}$ ) the relative out flowing particles above escape velocity normalized to $\mathrm{O}^{+}$, and (f and $\mathrm{l}$ ) the fraction of out flowing particles that has velocities above escaping velocity. All quantities are presented on log-scale except in panels $\mathrm{f}$ and 1 where linear scale is used.
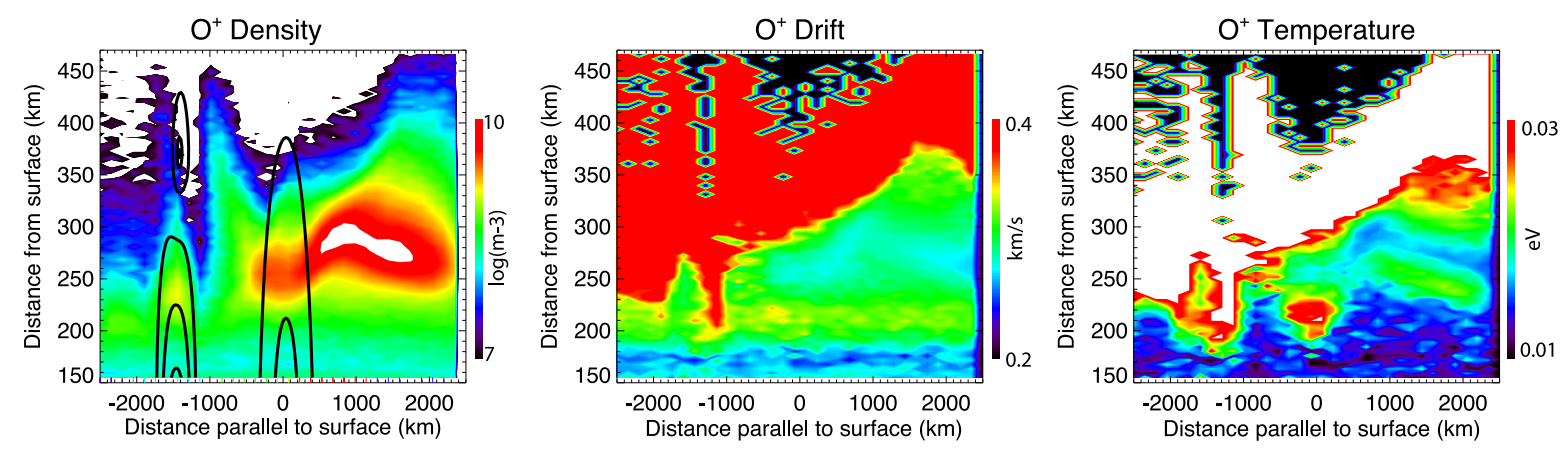

Fig. 6. Example of the $\mathrm{O}^{+}$density, drift and temperature for time 21:45 using Wind $\mathrm{B}$. The selected time is when the third crustal field region is within the simulations domain as indicated by the contour lines in the first panel of the total magnetic field. The color scale represent in the three panels density (log-scale), drift speed (linear-scale), and temperature (linear-scale, white area saturated). The drift and temperature numbers at high altitudes have large error bars due to low counting statistics.

The simulation results suggest that the fluctuations in the ion flow quantities are more sensitive to the moving crustal fields rather than the two wind profiles. But there are differences such as the timing of the ion outflow changes with respect to the different species. The relative outflows as function of ion species (Fig. 5 panels $d$ and $j$ and $e$ and $\mathrm{k}$ ) are to some degree influenced by the neutral wind, especially the $\mathrm{O}_{2}{ }^{+}$to $\mathrm{O}^{+}$ratio. The increase in the relative fraction of the outflow is observed to start first in $\mathrm{CO}_{2}{ }^{+}$then $\mathrm{N}_{2}{ }^{+}$and lastly $\mathrm{O}_{2}{ }^{+}$based on Fig. 5 (panels' $d$ and $\mathrm{j}$ and $\mathrm{e}$ and $\mathrm{k}$ ). The interpretation of timing and outflow rates in Fig. 5 has to be made with caution. 
Based on Fig. 5(g), the $\mathrm{O}_{2}{ }^{+}$outflow decreases slightly before $\mathrm{O}^{+}$, but the $\mathrm{O}^{+}$outflow decreases much more significantly leading to an increased ratio of $\mathrm{O}_{2}{ }^{+} / \mathrm{O}^{+}$(Fig. 5(j)). The ratio recovers when the $\mathrm{O}^{+}$loss starts to increase.

Most ions (created by photochemical reactions) are reabsorbed by photochemical reactions, as shown in Fig. 5(c) and 5(i). The photochemistry production to loss ratio is close to one indicating that the number of ions lost to space is a small fraction of the ions created within the simulation box's altitude region. For $\mathrm{O}_{2}^{+}$this ratio is closer to 2 explaining, to some degree, why it has much sharper fluctuations in the outflow rate compared to the others and why $\mathrm{O}_{2}{ }^{+}$has a long lifetime in the simulation domain. The reader should be reminded that Fig. 5 (panels $\mathrm{c}$ and i) depend on which altitude range is used, as the low altitude physics where the densities are high dominates the numbers for this ratio. The result suggests $\mathrm{O}_{2}{ }^{+}$is more dynamic in its outflow rate than for the other three ion species.

Lastly, the outflow fraction of escaping ions that have speeds above the escape speed is varying as presented in Fig. 5 (panels $\mathrm{f}$ and 1 ). $\mathrm{CO}_{2}{ }^{+}$and $\mathrm{N}_{2}{ }^{+}$have low scale heights and most of the ions leaving the simulation domain have speeds above the escape velocity. With increasing scale height the fraction can vary significantly with the crustal fields passing through the simulation domain. This effect is especially true for $\mathrm{O}^{+}$where the amplitude of this ratio increases with time (panels $f$ and $\mathrm{l}$ ) but the net outflow baseline (panels a and $g$ and $b$ and $h$ ) is constant. This result suggests that the velocity composition of $\mathrm{O}^{+}$strongly depends on the dynamics up to 10 hours prior (based on initially studies). Even though the lifetime of the ions are mainly less than $1 \mathrm{~h}$ it takes times to change the locations of the high-density regions explaining the long time constants in Mars ionosphere. The variations in global outflow will be investigated in future studies.

The neutral winds seem to not be important for the overall long-term ion outflow (Fig. 5) if the atmosphere has time to recover to the baseline (without crustal fields). However, neutral winds clearly affect the redistribution of the ions (Fig. 3). The $\mathrm{O}^{+}$density, drift, and temperature at $21 \mathrm{~h}$ 45 min are presented in Fig. 6. Black regions mean too low density for any statistical significance. Crustal field (limited to a single crustal field in the simulation domain) also seem to be unimportant to the long-term outflow. At low latitudes the drift speed reflects the neutral winds except where strong crustal fields are located. The highest drifts are located at high altitudes and are associated with the density gradients (i.e. in the region where the wave power is most efficient in heating each ion). Lastly, the ion temperature is not always monotonically increasing with altitude. As seen in Fig. 6 there are situations when the ion temperature is large below the peak density.

\section{Summary}

Recent MTGCM simulations of the upper atmosphere of Mars have suggested that significant neutral winds are common (Valeille et al., 2009). This paper evaluated the impact of neutral winds on the ion environment using the CAPIT code.

Based on the CAPIT simulation, it is clear that neutral winds can alter the distributions of the ions at Mars. The impact of neutral winds on the total ion outflow is, to first order, not important. Based on the presented simulations, changes in the magnetic field topology are more important for the short-term ion outflow rates than the neutral wind profile.

With significantly different neutral wind conditions, ion density, velocity, and temperature profiles will be significantly altered. With a satellite measuring the same location at two different times the ion profile can be quite different. It is therefore important to take into consideration neutral winds when interpreting low altitude satellite plasma observations. If the ion distribution can be measured well at low altitudes (i.e. below $\sim 250 \mathrm{~km}$ ), this can be used to infer the neutral wind direction and magnitude in the regions where ion-neutral collision is high.

Acknowledgments. This work was performed under the NASA Grant NNX08AN63G.

Guest editor M. Yamauchi thanks S. Bougher and an anonymous reviewer in evaluating this paper.

\section{References}

Andersson, L., R. E. Ergun, and A. I. F. Stewart, The combined atmospheric photochemistry and ion tracing code: reproducing the Viking Lander resul and initial outflow results, Icarus, 206, 120-129, 2009.

Bougher, S. W., P.-L. Blelly, M. Combi, J. L. Fox, I. Mueller-Wodarg, A. Ridley, and R. G. Roble, Neutral Upper Atmosphere and Ionosphere Modeling, Space Sci. Rev., 139, 107-141, doi:10.1007/s11214008-9401-9, 2008.

Brecht, S. H. and S. A. Ledvina, The solar wind interaction with the martian ionosphere/atmosphere, Space Sci. Rev., 126, 15-38, 2006.

Carlsson, E., A. Fedorov, S. Barabash, and 44 other coauthors, Mass composition of the escaping plasma at Mars, Icarus, 182(2), 320-328, 2006.

Dubinin, E., M. Franz, J. Woch, E. Roussos, S. Barabash, R. Lundin, J. D. Winningham, R. A. Frahm, and M. Acuna, Plasma morphology at Mars, Aspera-3 observations, Space Sci. Rev., 126, 209-238, 2006.

Duru, F., D. A. Grunett, D. D. Morgan, R. Modolo, A. F. Nagy, and D. Najib, Electron densities in the upper ionosphere of Mars from the excitation of electron plasma oscillations, J. Geophys. Res., 113, A07302, doi:10.1029/2008JA013073, 2008.

Espley, J. R., P. A. Cloutier, D. A. Brain, D. H. Crider, and M. H. Acuña, Observations of low frequency magnetic oscillations in the martian magnetosheath, magnetic pileup region, and tail, J. Geophys. Res., 109, A07213, 2004.

Fox, J. L., Upper limits to the outflow of ions at Mars: Implications for atmospheric evolution, Geophys. Res. Lett., 24, 2901-2904, 1997.

Fox, J. L. and K. E. Yeager, Morphology of the near-terminator martian ionosphere: A comparison of models and data, J. Geophys. Res., 111, A10309, 2006.

Haider, S. A., $\mathrm{O}^{+}$escape in the polar ion exosphere of Mars, Adv. Space Res., 16, 49-55, 1995.

Hanson, W. B. and G. P. Mantas, Viking electron temperature measurements: Evidence for a magnetic field in the martian ionosphere, J. Geophys. Res., 93, 7538-7544, 1988.

Hanson, W. B., S. Sanatani, and D. R. Zuccaro, The martian ionosphere observed by the Viking retarding potential analyzer, J. Geophys. Res., 82, 4351-4363, 1977.

Kallio, E., K. Liu, R. Jarvinen, V. Pohjola, and P. Janhunen, Oxygen ion escape at Mats in a hybrid model: High energy and low energy ions, Icarus, 206, 152-163, 2010.

Lammer, H., H. I. M. Lichtenegger, C. Kolb, I. Ribas, E. F. Guinan, R. Abart, and S. J. Bauer, Loss of water from Mars: Implications for the oxidation of the soil, Icarus, 165, 9-25, 2003.

Lundin, R. and 27 colleagues, Ionospheric plasma acceleration at Mars: ASPERA-3 results, Icarus, 182, 308-319, 2006.

Lundin, R., S. Barabash, M. Holmstrom, H. Nilsson, M. Yamauchi, E. M. Dubini, and M. Fraenz, Atmospheric origin of cold ion escape from Mars, Geophys. Res. Lett., 36, doi:10.1029/2009GL039341, 2009.

Ma, Y.-J. and A. F. Nagy, Ion escape fluxes from Mars, Geophys. Res. Lett., 
34, L08201, 2007.

Ma, Y.-J., A. F. Nagy, I. V. Sokolov, and K. C. Hansen, Three-dimensional, multispecies, high spatial resolution MHD studies of the solar wind interaction with Mars, J. Geophys. Res., 109, A07211, 2004.

Mitchell, D. L., R. P. Lin, C. Mazelle, H. Reme, P. A. Cloutier, J. E. P. Connerney, M. H. Acuña, and N. F. Ness, Probing Mars' crustal magnetic field and ionosphere with the MGS Electron Reflectometer, J. Geophys. Res., 106, 23419-23427, 2001.

Modolo, R., G. M. Chanteur, E. Dubinin, and A. P. Matthews, Influence of the solar EUV flux on the martian plasma environment, Ann. Geophys., 23, 433-444, 2005.

Nair, H., M. Allen, A. D. Anbar, and Y. L. Yung, A photochemical model of the martian atmosphere, Icarus, 111, 124-150, 1994.

Nairn, C. M. C., R. Gard, A. Skalsky, and J. G. Trotignon, Plasma wave observations in the night sector of Mars, J. Geophys. Res., 96, 1122711233, 1991.

Nier, A. O. and M. B. McElroy, Composition and structure of Mars' upper atmosphere: Results from the Neutral Mass Spectrometers on Viking 1 and 2, J. Geophys. Res., 82, 4341-4349, 1977.

Nilsson, H., E. Carlsson, D. A. Brain, M. Yamauchi, M. Holmstron, S.
Barabash, R. Lundin, and Y. Futaana, Ion escape form Mars as a function of solar wind conditions: A statistical study, Icarus, 206, 40-49, 2010

Terada, N., Y. N. Kulikov, H. Lammer, I. M. Lichtenegger, T. Tanaka, H. Shinagawa, and T. Zhang, Atmospheric and water loss from early Mars under extreme solar wind and extreme ultraviolet conditions, Astrobiology, 9, 55-70, 2009.

Valeille, A., V. Tenishev, S. W. Bougher, M. R. Combi, and A. F. Nagy, Three-dimensional study of Mars upper thermosphere/ionosphere and hot oxygen corona: 1. General description and results at equinox for solar low conditions, J. Geophys. Res., 114, E11005, doi:10.1029/2009JE003388, 2009.

Winningham, J. D., R. A. Frahm, J. R. Sharber, and 43 other coauthors, Electron oscillations in the induced martian magnetosphere, Icarus, 182(2), 360-370, 2006.

L. Andersson (e-mail: Laila.Andersson@1asp.colorado.edu) and R. E. Ergun 